\title{
МОДЕЛЬ СКОРОСТИ ВЕТРА НА ОСНОВЕ ДРОБНОГО СТОХАСТИЧЕСКОГО ПРОЦЕССА
}

\author{
Давыдов Денис Юрьевич1, \\ denisyudavydov@gmail.com
}

\author{
Обухов Сергей Геннадьевич ${ }^{1}$, \\ serob99@mail.ru \\ 1 Национальный исследовательский Томский политехнический университет, \\ Россия, 634050, г. Томск, пр. Ленина, 30.
}

\begin{abstract}
Актуальной проблемой развития и повышения конкурентоспособности нефртегазовой отрасли является решение задачи надежного и эфффективного электроснабжения технологических объектов новых месторождений и магистральных нефртегазопроводов, территориально удаленных от центральной электрической сети. Одним из эфрфективных способов решения данной проблемы является применение ветроэнергетических установок. Первоочередной задачей проектирования и технико-экономического обоснования применения ветроэнергетических установок является прогноз изменения скоростей ветра в месте установки электростанции. Стохастическая природа ветра и его большая изменчивость во времени и пространстве определяют высокую сложность данной задачи, для решения которой используют методы математического моделирования. Известные модели скорости ветра на основе цепей Маркова, авторегрессии и скользящего среднего не позволяют осуществлять варьирование шага моделирования, что ограничивает возможность их применения для имитационного моделирования режимов ветроэнергетических установок и энергетических систем на их основе. В статье предложена модель скорости ветра на основе стохастического диффреренциального уравнения, устраняющая данный недостаток.

Цель: разработка модели скорости ветра на основе стохастического диффреренциального уравнения дробного процесса Орнштейна-Уленбека с периодической функцией среднего значения, обеспечивающей моделирование статических и динамических режимов работы ветроэнергетической установки на различных временных интервалах.

Mетоды: математическое и компьютерное моделирование с использованием программной среды MatLab/Simulink.

Результаты. Предложенная модель, в отличие от известных моделей подобного типа, воспроизводит как суточные, так и сезонные вариации скорости ветра, а также долговременную корреляционную зависимость моделируемого процесса, что позволяет осуществлять моделирование рабочих режимов ветроэнергетических установок на различных временных интервалах с требуемой дискретизацией. Валидация модели выполнена с использованием данных климатических наблюдений скорости ветра из электронного архива Всероссийского института гидрометеорологической информации. Адекватность модели подтверждена хорошей степенью соответствия моделируемых траекторий скорости ветра с данными фактических наблюдений, зарегистрированных на 518 метеостанциях, расположенных на территории России.
\end{abstract}

\section{Ключевые слова:}

Ветроэнергетика, моделирование скорости ветра, стохастические диффференциальные уравнения, дробное броуновское движение, временные ряды.

\section{Введение}

В настоящее время большая часть бюджета РФ обеспечивается за счет доходов от продажи нефти и газа, что определяет ведущую роль нефтегазовой промышленности в социально-экономическом развитии страны. Для развития нефтегазовой отрасли необходимо освоение новых месторождений и строительство магистральных нефтегазопроводов, которые преимущественно располагаются в районах, удаленных от центральной электрической сети. Надежное и эффективное электроснабжение технологических объектов децентрализованных регионов является важной и актуальной задачей нефтегазовой промышленности, что определяет высокие требования к надежности систем электроснабжения. Известные стандартные проекты систем электроснабжения не всегда отвечают установленным требованиям надежности, что требует применения новых технических решений. Одним из таких решений является применение в системах электроснабжения ветроэнергетических установок (ВЭУ).

Положительный опыт применения оффшорных ветроэлектростанций для электроснабжения нефтя- ных шельфовых месторождений приведен в работе [1], технология термической интенсификации добычи нефти, основанная на применении ВЭУ, описана в работе [2], высокая оценка перспектив применения ВЭУ для энергообеспечения объектов газовой промышленности получена в работах [3, 4]. Результаты проведенных исследований доказывают, что применение ВЭУ в системах электроснабжения технологических объектов нефтегазовой промышленности обеспечивает снижение энергоемкости и себестоимости добываемых георесурсов, а также повышает экологическую и энергетическую безопасность в районах децентрализованного энергоснабжения.

Для решения задач технико-экономического обоснования и проектирования энергетических систем с применением ВЭУ необходим достоверный прогноз изменения скоростей ветра на коротких и длительных временных интервалах. Стохастическая природа ветра и его большая изменчивость во времени и пространстве определяют высокую сложность данной задачи, для решения которой используют методы математического моделирования. Скорость ветра моделируется как стационарный стохастический процесс, характеристиками которого являются: 
- функция плотности вероятности (Probability Density Function - PDF), описывающая статистическое распределение скоростей ветра. Данная характеристика используется в большинстве ветроэнергетических расчетов для оценки потенциальной выработки энергии;

- функция автокорреляции (Autocorrelation Function - ACF), характеризующая степень взаимосвязи между последовательными значениями временного ряда [5] и обеспечивающая повышение точности моделирования флуктуаций скорости ветра на коротких временных интервалах;

- нестационарные суточные и сезонные вариации [6], которые значительно влияют на производительность ВЭУ [7] и моделирование которых необходимо для прогнозирования выработки электроэнергии [8], анализа аэродинамического взаимовлияния между ВЭУ, работающими в составе ветропарков [9], и согласования режимов работы основного генерирующего оборудования гибридных энергетических систем на основе возобновляемых источников энергии (ВИЭ).

Дискретные модели временных рядов, к которым относятся модели авторегрессионного скользящего среднего, модели на основе цепей Маркова [10-12], имеют недостаток, связанный с тем, что не позволяют осуществлять варьирование шага моделирования. Это ограничение исключает возможность применения данных моделей для имитационного моделирования режимов ВЭУ и энергетических систем на их основе с высокой степенью временной дискретизации, что является необходимым условием для решения задач выбора состава генерирующего оборудования, анализа установившихся и переходных процессов проектируемых электроэнергетических систем.

В качестве альтернативы вышеперечисленным методам в настоящее время предложены модели на основе стохастических дифференциальных уравнений (Stochastic Differential Equations - SDE), которые позволяют масштабировать во времени моделируемый процесс с сохранением его статистических характеристик. Методы моделирования скорости ветра на основе данного подхода описаны в работах [13-15]. Модели $[13,14]$ позволяют синтезировать временной ряд скорости ветра с заданным статистическим распределением и экспоненциально затухающей автокорреляцией. Прогнозирование суточного цикла скорости ветра реализовано в модели [15], однако возможность моделирования месячных вариаций не предусмотрена. Кроме этого, в предложенных моделях стохастическая составляющая скорости ветра генерируется посредством SDE на основе винеровского процесса (случайных приращений), что не позволяет воспроизвести долговременную корреляционную зависимость, которая характерна для временных рядов метеорологических переменных [6].

Целью данной работы является разработка модели скорости ветра на основе стохастического дифференциального уравнения, обеспечивающей имитационное моделирование режимов работы ВЭУ на различ- ных временных интервалах. Основой для построения такой модели послужила методика моделирования скорости ветра на основе SDE процесса ОрнштейнаУленбека [16], описанная в работах [13, 14]. Учитывая факт того, что временные ряды метеорологических переменных имеют признаки долговременной корреляционной зависимости и сезонные периодические составляющие, в качестве более подходящего решения предлагается использовать SDE процесс Орнштейна-Уленбека на основе дробного броуновского движения (Fractional Brownian Motion - FBM) и периодической функции среднего значения.

\section{Описание методов и алгоритмов}

Предложенный алгоритм моделирования траекторий скорости ветра состоит из трех этапов: калибровки модели (оценки параметров) на основе данных временного ряда долгосрочных наблюдений, численного моделирования траекторий скорости ветра и преобразования распределения полученной автокоррелированной последовательности в распределение, соответствующее данным реальных наблюдений. Блок-схема алгоритма моделирования приведена на рис. 1.

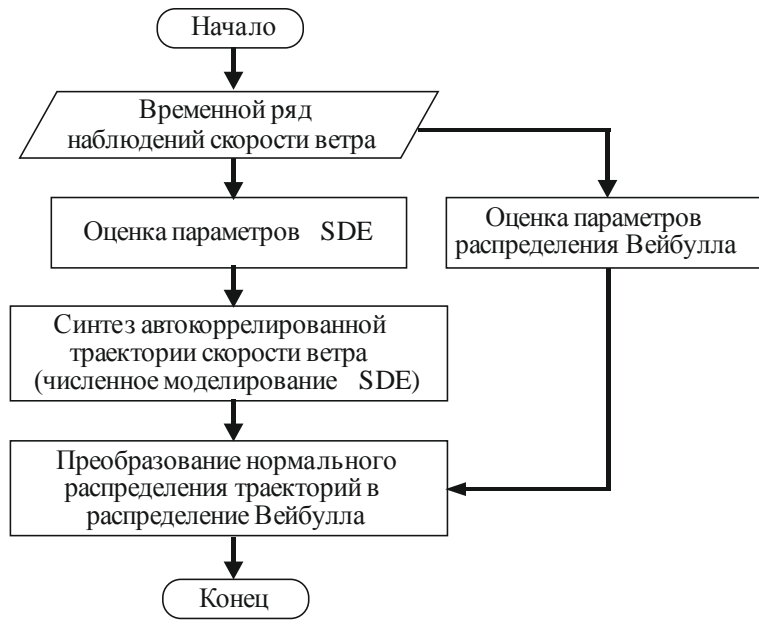

Рис. 1. Блок-схема алгоритма моделирования скорости ветра

Fig. 1. Wind speed simulation algorithm flowchart

Стохастическое дифференциальное уравнение дробного процесса Орнштейна-Уленбека может быть записано в следующей форме:

$$
d X_{t}=\theta\left(\mu-X_{t}\right) d t+\sigma d W_{t}^{H},
$$

где $X_{t}$ - мгновенное значение моделируемой переменной процесса в момент времени $t ; \theta$ - параметр скорости возврата к среднему (равновесному) уровню $(\mu) ; \sigma$ - параметр дисперсии приращений; $W_{t}^{H}-$ дробное броуновское движение (FBM).

FBM представляет собой непрерывный центрированный гауссовский процесс со стационарными приращениями и ковариационной функцией следующего вида [17]:

$$
E\left(W_{t}^{H}, W_{s}^{H}\right)=\frac{1}{2}\left(|s|^{2 H}+|t|^{2 H}-|t-s|^{2 H}\right),
$$


где $s$ - шаг времени, предшествующий текущему шагу $t ; H \in(0,1)$ - показатель Херста, характеризующий степень самоподобия приращений и тип памяти процесса.

При $H=1 / 2$ FBM вырождается в стандартное броуновское движение, приращения которого независимы (Винеровский процесс), и в таком случае функция автокорреляции стохастического процесса, описываемого уравнением (1), представляет собой экспоненциально убывающую характеристику. Данный процесс является Марковским и не обладает свойством долгой памяти, что в свою очередь не позволят воспроизвести характерную для временных рядов метеорологических данных долговременную корреляционную зависимость. При $H>1 / 2$ приращения имеют положительную корреляцию и АCF процесса (1) имеет вид степенной функции, что в большей степени соответствует $\mathrm{ACF}$ временных рядов с долговременной корреляционной зависимостью.

В своей исходной форме уравнение процесса (1) позволяет смоделировать только стационарную стохастическую составляющую временного ряда с автокорреляционной зависимостью, скорость и характер затухания которой задаются параметрами $H$ и $\theta>0$.

Для моделирования нестационарной составляющей, обусловленной циклическими изменениями скорости ветра в течение суток, используется уравнение процесса (1) с периодической функцией среднего значения [18]:

$$
\begin{gathered}
y(t)=\alpha \cos \left(\frac{2 \pi\left(t-t_{\text {peak }}\right)}{24}\right), \\
d X_{t}=\left(\frac{d y(t)}{d t}+\theta\left[y(t)-X_{t}\right]\right) d t+\sigma d W_{t}^{H},
\end{gathered}
$$

где $y(t)$ - функция, моделирующая цикл изменения скорости ветра в момент времени $(t) ; \alpha$ - параметр амплитуды; $t_{\text {peak }}$ - время суточного максимума скорости ветра.

В такой форме уравнение (3) описывает процесс $X$, отклоняющийся от детерминированной траектории циклически изменяющегося среднего уровня, определяемого в каждый момент времени функцией $y(t)$, и возвращающийся к нему со скоростью, пропорциональной отклонению.

Моделирование траекторий стохастического процесса, описываемого уравнением (3), выполняется с использованием схемы Эйлера-Маруямы, подходящей для численного моделирования SDE процессов с длительной памятью $(H \geq 1 / 2)$ [19]. Схема дискретизации SDE (3) записывается следующим образом:

$$
\begin{aligned}
\hat{X}_{n+1}=\hat{X}_{n}+ & {\left[d y\left(t_{n}\right)+\hat{\theta}\left(y\left(t_{n}\right)-\hat{X}_{n}\right)\right] \Delta t+} \\
& +\hat{\sigma}\left(W_{n+1}^{H}-W_{n}^{H}\right),
\end{aligned}
$$

где $\Delta t$ - шаг расчета; $t$ - время моделирования; $W^{H}$ дробный гауссовский шум с показателем Херста $(H>1 / 2)$.

В процессе моделирования для генерирования дробного гауссовского шума $W^{(H>1 / 2)}$ используется метод циркулянтного вложения [20].
Реализации стохастического процесса будут иметь нормальное распределение, что не характерно для реальных данных наблюдений скорости ветра, статистическое распределение которых на практике чаще всего аппроксимируется двухпараметрическим распределением Вейбулла [21]. Основываясь на методике [13], для получения последовательности с заданным распределением применяется метод обратного преобразования:

$$
V=F^{-1}[\Phi(X)]
$$

где $\Phi(\cdot)$ - кумулятивная функция (Cumulative Distribution Function - CDF) нормального распределения; $F^{-1}$ - обратная функция требуемого распределения.

Моделирование сезонной составляющей выполняется путем последовательного преобразования переменных сгенерированного ряда $X(t)$ в переменные распределения Вейбулла $V(t)$ с параметрами $(c, k)$, определенными для каждого месяца:

$$
V(t)=c_{\mathrm{s}}(t)[-\ln (1-\Phi[X(t)])]^{\left(1 / k_{s}(t)\right)},
$$

где $V(t)$ - преобразованный процесс с заданным распределением; $c_{\mathrm{s}}, k_{\mathrm{s}}$ - параметры масштаба и формы распределения Вейбулла, определенные для каждого месяца года $(\mathrm{s}=1,2 \ldots 12)$.

\section{Оценка параметров модели}

В процессе калибровки модели требуется оценка следующих параметров: показателя Херста, параметра скорости возврата к среднему, дисперсии приращений, параметров амплитуды и времени суточного максимума скорости ветра, а также параметров распределения. Оценка параметров осуществляется по выборочным данным временного ряда наблюдений скорости ветра. На первом этапе выполняется оценка параметров модели распределения, для чего выборка исходных данных разделяется по месяцам на 12 групп, и для каждой группы определяются оценки параметров распределения Вейбулла.

Перед оценкой остальных параметров модели выполняется нормализация ряда выборочных данных таким образом, чтобы средневыборочное значение и дисперсия были эквивалентны математическому ожиданию и дисперсии стохастического процесса (т. е. $\left.\mu=0, \sigma^{2}=1\right)$. Кроме этого, из данных временного ряда устраняется сезонная (месячная) компонента.

\section{Оценка показателя Херста}

В данном исследовании для оценки показателя Херста использовался метод агрегированной дисперсии [22]. Выборочное множество данных $X=\left\{X_{i}, i \geq 1\right\}$ разделяется на блоки $(k)$ с числом элементов $(m)$, и для каждого блока рассчитывается среднее значение:

$$
X^{(m)}(k)=\frac{1}{m} \sum_{i=(k-1) m+1}^{k m} X(i) \quad k=1,2 \ldots N / m,
$$

где $N$ - общее число элементов выборки.

Поскольку дисперсия дробного гауссовского шума равна $\operatorname{Var} X^{(m)}=\sigma_{0} m^{\beta}$ при $m \rightarrow \infty$, где $\beta=2 H-2<0$, точечной оценкой $\operatorname{Var}^{(m)}$ является дисперсия выборки, ко- 
торая рассчитывается для нескольких значений $(m)$ следующим образом:

$$
\overline{\operatorname{Var}} X^{(m)}=\frac{1}{N / m} \sum_{k=1}^{N / m}\left(X^{(m)}(k)\right)^{2}-\left(\frac{1}{N / m} \sum_{k=1}^{N / m} X^{(m)}(k)\right)^{2} .
$$

Расчетные значения $(m)$ выбираются таким образом, чтобы точки $\log (m)$ на графике зависимости $\log \left(\overline{\operatorname{Var}} X^{(m)}\right)$ от $\log (m)$ были равноудалены друг от друга, т. е. $m_{i+1} / m_{i}=C$, где $C-$ константа, зависящая от размера выборки и желаемого количества точек. Тогда зависимость $\log \left(\overline{\operatorname{Var}} X^{(m)}\right)$ от $\log (m)$ может быть аппроксимирована прямой, угол наклона которой равен $(\beta)$ и оценка показателя Херста определена как

$$
H=\frac{\beta+2}{2} \text {. }
$$

\section{Оценка параметров SDE}

Оценка параметра дисперсии $(\sigma)$ стохастического процесса может быть вычислена по квадратической вариации выборочных данных временного ряда по следующей формуле [23]:

$$
\hat{\sigma}^{2}=\frac{\sum_{i=1}^{n-1}\left(X_{(i+1)}-X_{i}\right)^{2}}{(N-1) \Delta t^{2 \hat{H}}},
$$

где $X_{i}, X_{(i+1)}$ - значения временного ряда на $i$-м и последующем шаге времени; $\Delta t-$ интервал дискретизации выборки; $H$ - оценка показателя Херста.

Поскольку скорость ветра можно рассматривать как стационарный процесс с долговременной зависимостью $(H>1 / 2$ и $\theta>0)$, оценка параметра скорости возврата к среднему $(\theta)$ может быть получена из следующего выражения [24]:

$$
\hat{\theta}=\left[\frac{1}{\hat{\sigma}^{2} \hat{H} \Gamma(2 \hat{H}) N} \sum_{i=1}^{N} X_{i}^{2}\right]^{-\frac{1}{2 H}},
$$

где $\Gamma$ - гамма-функция.

Оценка параметров периодической функции, моделирующей суточный ход скорости ветра

Вычисление параметров амплитуды и времени максимума суточного цикла скорости ветра осуществляется путем подгонки кривой функции (2) к точкам фактического суточного профиля (Diurnal Profile - DP), значения которых для каждого срока наблюдения вычисляются по следующей формуле:

$$
D P(\tau)=\frac{1}{N / n_{d}} \cdot \sum_{i=0}^{\left(N / n_{d}\right)-1} X_{\left(i \cdot n_{d}\right)+\tau}, \tau=1,2 \ldots n,
$$

где $N$ - число элементов выборки; $n_{d}$ - число сроков наблюдения в течение суток; $\tau$ - порядковый номер срока наблюдения.

В ходе тестирования модели выявлена необходимость нормализации значений суточного профиля перед выполнением аппроксимации, что осуществляется следующим образом:

$$
D P^{\prime}=\frac{D P}{\left(\frac{\sigma_{X}}{\hat{\sigma}}-\frac{1}{2}\right)},
$$

где $\sigma_{\mathrm{X}}-$ квадратичное отклонение выборочных данных.

\section{Оценка адекватности модели}

Алгоритм проверки адекватности моделирования состоит в оценке степени соответствия характеристик синтезированных и фактических данных временных рядов скорости ветра. В исследовании используются данные долгосрочных наблюдений скорости ветра, зарегистрированные на 518 метеостанциях России [25]. Временные ряды содержат данные восьмисрочных наблюдений, зафиксированных в стандартные синоптические сроки с интервалом времени в 3 часа. Карта расположения метеостанций показана на рис. 2.

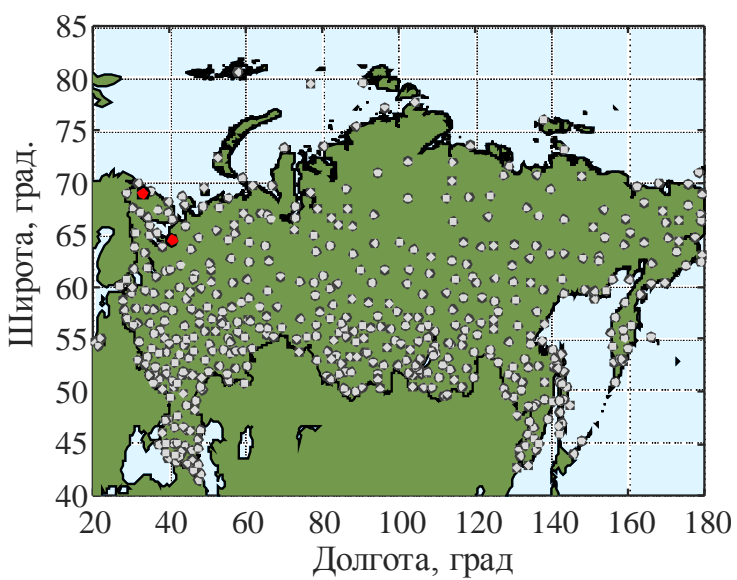

Pис. 2. Карта расположения метеостанций

Fig. 2. Weather station location map

Оценки параметров модели определены для выборочных данных временных рядов скорости ветра каждой метеостанции, статистические распределения оценок параметров показаны на диаграмме, изображенной на рис. 3.

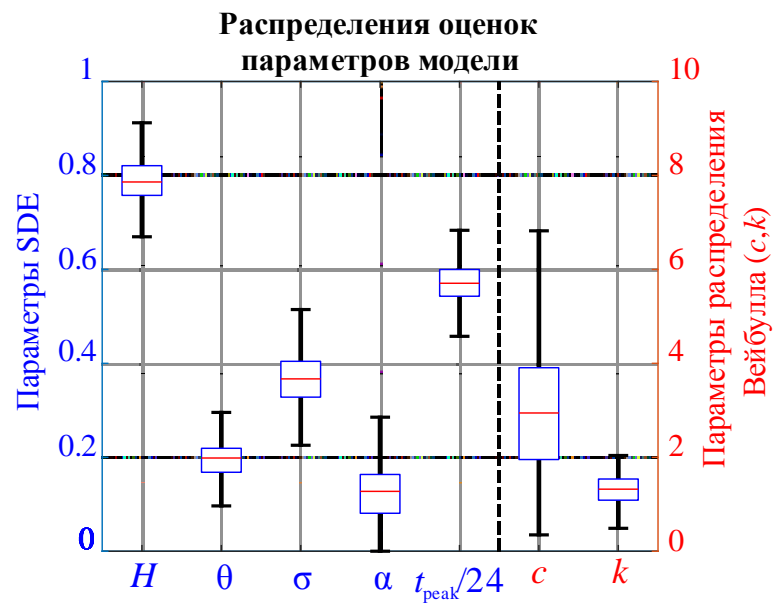

Рис. 3. Диаграмма распределения оченок параметров модели

Fig. 3. Boxplot of model parameters estimates 
Моделирование траектории скорости ветра выполнялось на временном интервале, равном 20 годам, с шагом дискретизации, равным исходному интервалу между наблюдениями ( $\Delta t=3$ часа). Для синтезированных временных рядов скорости ветра рассчитаны коэффициенты последовательной автокорреляции между значениями, автокорреляции среднемесячных значений, суточные профили и гистограммы статистического распределения. Полученные характеристики сравниваются с характеристиками исходного временного ряда, и их степень соответствия определяется по величине коэффициента детерминации [26]:

$$
R^{2}=1-\frac{\sum\left(y_{i}-f_{i}\right)^{2}}{\sum\left(y_{i}-\bar{y}_{i}\right)^{2}},
$$

где $y_{i}$ - характеристика фактического временного ряда данных; $f_{i}$ - характеристика синтезированного временного ряда.

Расчет относительной ошибки между оценками показателя Херста синтезированных $\left(\hat{H}_{Y}\right)$ и фактических $\left(\hat{H}_{X}\right)$ временных рядов выполнен по формуле:

$$
\delta \hat{H}=\frac{\left|\hat{H}_{Y}-\hat{H}_{X}\right|}{\hat{H}_{X}} \cdot 100 .
$$

В ходе тестирования выполнено сравнение стандартной модели с независимыми приращениями $(H=1 / 2)$ и модели на основе дробного процесса с долговременной корреляционной зависимостью $(H>1 / 2)$. Для сравнения используется Байесовский информационный критерий (Bayesian information criterion BIC) [27]:

$$
B I C=-2 \ln (L)+\ln (n) k,
$$

где $L$ - максимальное значение функции правдоподобия; $k$ - количество оцененных параметров модели; $n$ - число элементов временного ряда.

\section{Результаты исследования}

Далее в качестве примера приводятся результаты сравнения характеристик смоделированных траекторий SDE с характеристиками временных рядов фактических данных, зарегистрированных на метеостанциях Мурманска и Архангельска. Международные номера этих станций в списке Всемирной метеорологической организации, а также основные характеристики их месторасположения приведены в табл. 1.

На рис. 4 показаны автокоррелограммы выборочных данных скорости ветра оригинальных и синтезированных временных рядов с максимальным сдвигом по времени равным 120 часам. Можно видеть, что при моделировании скорости ветра посредством SDE процесса без долговременной зависимости $(H=1 / 2)$
$\mathrm{ACF}$ значений синтезированного временного ряда согласуется только с первым коэффициентом эмпирической ACF, а затем убывает практически до нулевого значения. В то же время ACF модели процесса с длительной памятью $(H>1 / 2)$ имеет степенной характер убывания, в большей степени соответствующий эмпирической характеристике, что подтверждается высоким показателем коэффициента $R^{2}>90 \%$.

Таблица 1. Основные сведения о метеостанциях

Table 1. General information about weather stations

\begin{tabular}{|c|c|c|c|c|}
\hline $\begin{array}{c}\text { Номер } \\
\text { Number (ID) }\end{array}$ & $\begin{array}{c}\text { Название } \\
\text { Name }\end{array}$ & $\begin{array}{c}\text { Широта, } \\
\text { Latitude, }\end{array}$ & $\begin{array}{c}\text { Долгота, } \\
\text { Longitude, }\end{array}$ & $\begin{array}{c}\text { Высота, м } \\
\text { Elevation, m }\end{array}$ \\
\hline 22113 & $\begin{array}{c}\text { Мурманск } \\
\text { Murmansk }\end{array}$ & 68,97 & 33,05 & 57 \\
\hline 22550 & $\begin{array}{c}\text { Apхангельск } \\
\text { Arkhangelsk }\end{array}$ & 64,50 & 40,73 & 8 \\
\hline
\end{tabular}

Периодические осцилляции автокорреляционных функций обусловлены наличием циклической (суточной) составляющей. На рис. 5 продемонстрированы суточные профили скорости ветра для каждого срока наблюдений, которые аппроксимированы аналитической функцией $y(t)(2)$. Можно видеть, что суточные профили, полученные посредством моделирования (Model), с достаточной точностью повторяют форму кривой данных траекторий и хорошо согласуются с эмпирическими данными, о чем свидетельствует высокий показатель коэффициента детерминации $\left(R^{2}>0,95\right)$.

Автокоррелограммы среднемесячных значений скорости ветра, изображенные на рис. 6 отчетливо демонстрируют наличие повторяющейся сезонной компоненты. Наиболее значимая корреляция между летними и зимними периодами наблюдается на ACF данных метеостанции Мурманска. В то же время для данных метеостанции Архангельска данная связь носит менее выраженный характер. Результаты моделирования демонстрируют способность модели воспроизвести данную закономерность с приемлемой степенью точности $\left(R^{2}>70 \%\right)$.

На рис. 7 показаны гистограммы фактического распределения скорости ветра, аппроксимированного функцией распределения Вейбулла (Weibull), а также кривые плотности распределения вероятности синтезированных временных рядов (Model). Можно видеть, что используемый подход преобразования нормального распределения стохастического процесса в распределение Вейбулла позволяет получить последовательности, хорошо согласующиеся с распределением фактических данных.

Результаты оценки достоверности моделирования, проведенной по вышеописанной методике, для каждого из 518 временных рядов данных, используемых в исследовании, сведены в табл. 2. 

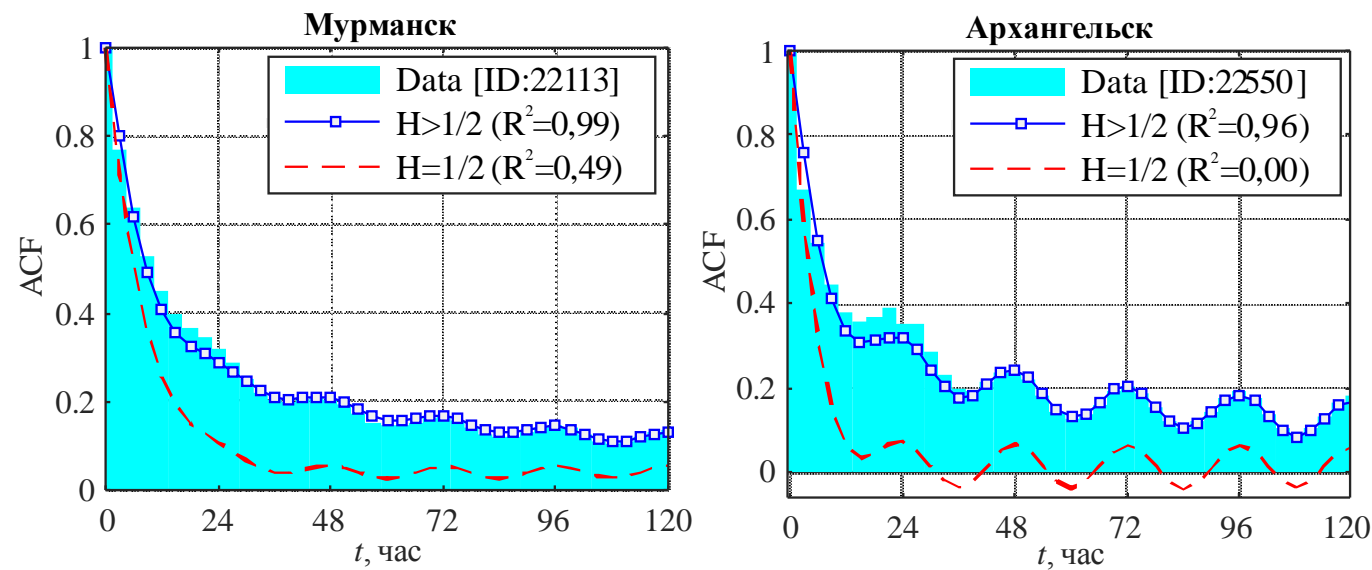

Рис. 4. Автокорреляция фактических и синтезированных временных рядов скорости ветра

Fig. 4. Autocorrelation of observed and simulated wind speed time series
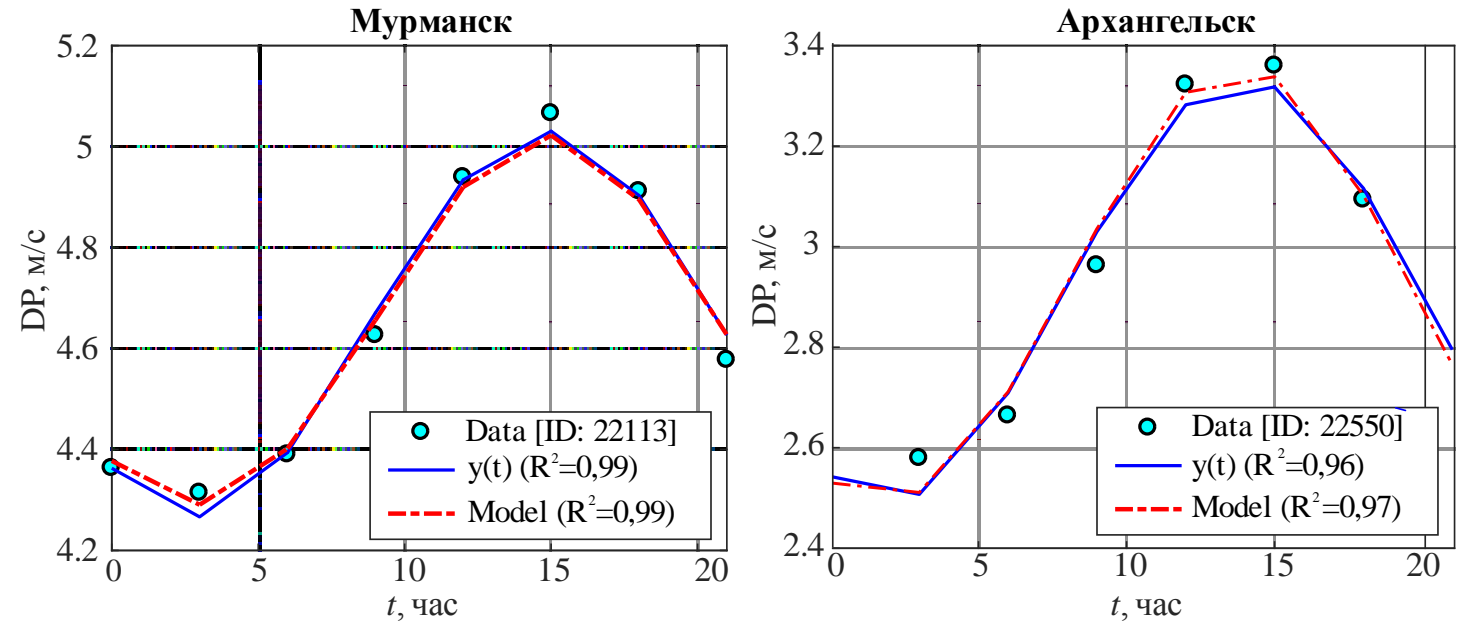

Pис. 5. Суточный профиль скорости ветра

Fig. 5. Wind speed diurnal profile
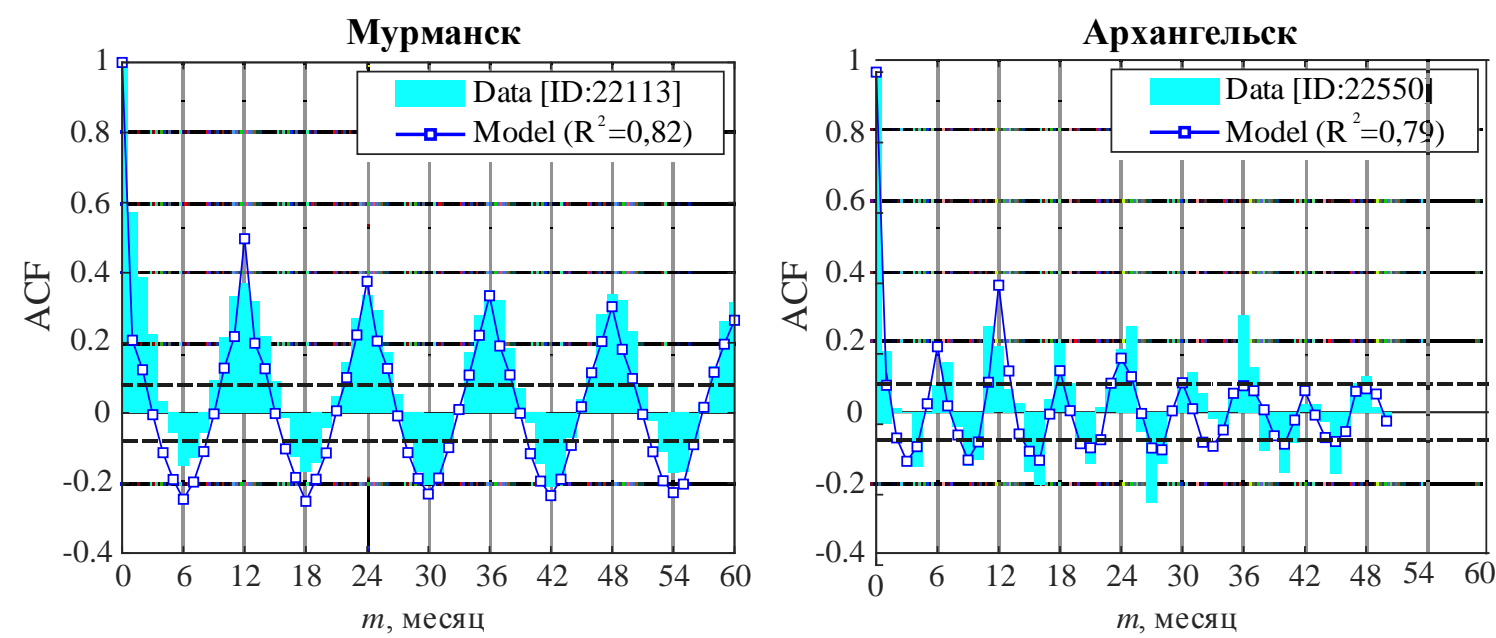

Рис. 6. Автокорреляция среднемесячных значений скорости ветра

Fig. 6. Autocorrelation of monthly-averaged wind speed 

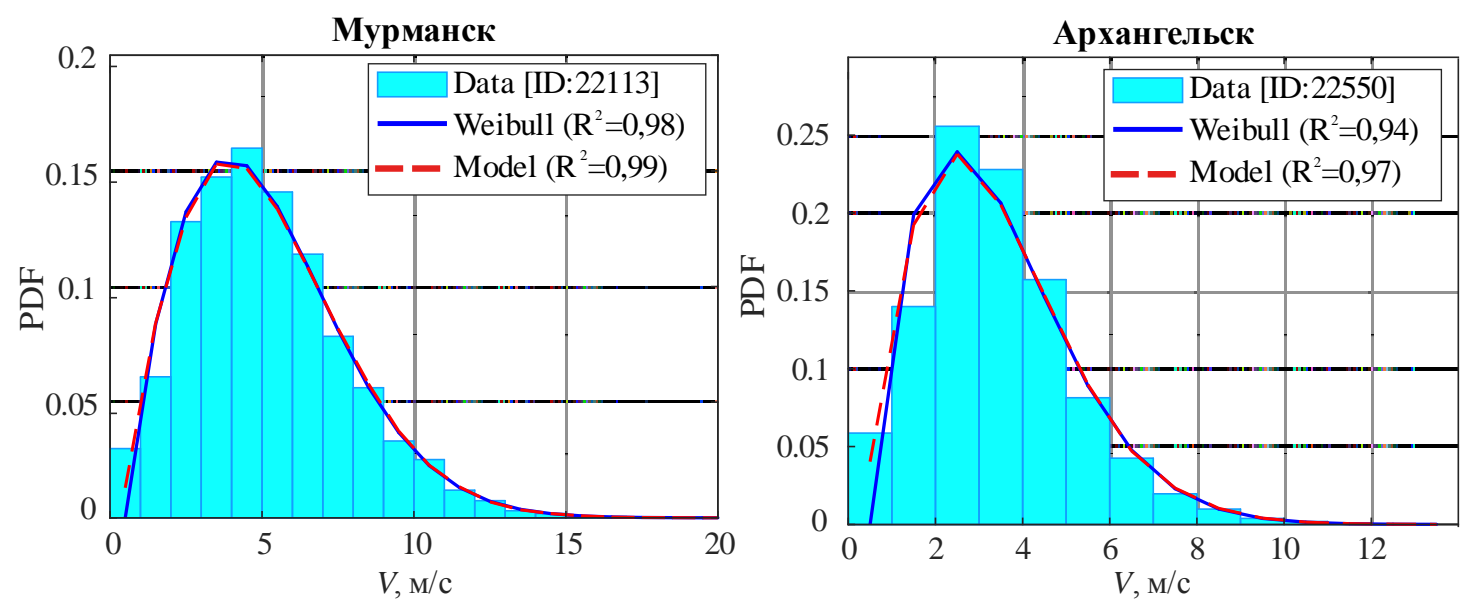

Pис. 7. Распределение фактических и смоделированных данных скорости ветра

Fig. 7. Distributions of observed and simulated wind speed data

Таблица 2. Результаты сравнения моделей

Table 2. Model comparison results

\begin{tabular}{|c|c|c|}
\hline $\begin{array}{c}\text { Критерий } \\
\text { Criterion }\end{array}$ & $\begin{array}{c}\text { Стандартная модель } \\
\text { Standard model } \\
(H=1 / 2)\end{array}$ & $\begin{array}{c}\text { Дробная модель } \\
\text { Fractional model } \\
(H>1 / 2)\end{array}$ \\
\hline$k$ & 28 & 29 \\
\hline BIC & 3,2267 & 3,2160 \\
\hline$R^{2}(\mathrm{ACF})$ & 0,3190 & 0,9322 \\
\hline$R^{2}(\mathrm{DP})$ & 0,9097 & 0,9160 \\
\hline$R^{2}(\mathrm{PDF})$ & 0,9215 & 0,9217 \\
\hline$\delta \hat{H}, \%$ & 10,042 & 1,3644 \\
\hline
\end{tabular}

Исходя из того, что относительное качество модели определяется меньшим значением показателя ВIC, можно сделать вывод, что вариант использования дробного стохастического процесса для моделирования динамики скорости ветра является более подходящим с точки зрения погрешности и количества параметров модели. Различие в показателях достоверности моделирования суточного профиля и статистического распределения для сравниваемых моделей несущественно.

\section{СПИСОК ЛИТЕРАТУРЫ}

1. Choi Y., Lee C., Song J. Review of renewable energy technologies utilized in the oil and gas industry // International Journal of Renewable Energy Research. - 2017. - V. 7. - № 2. - P. 592-598.

2. Бельский А.А., Климко В.И. Интенсификация добычи нефти. Концепция теплового метода с применением автономных ветроэлектрических установок // Деловой журнал «Neftegaz.RU». - 2016. - № 1-2. - C. 38-41.

3. Горюнов О.А., Назарова Ю.А. Перспективы применения ветроэнергетических установок для энергообеспечения объектов газовой промышленности в районах Крайнего Севера // Территория «НЕФТЕГАЗ». - 2015. - № 12. - С. 146-150.

4. Васенин А.Б., Крюков О.В. Вопросы электропитания вдольтрассовых объектов Единой системы газоснабжения России // Научно-технический сборник «Вести газовой науки». 2020. - № 2 (44). - C. 181-192.

5. Brett A.C., Tuller S.E. The autocorrelation of hourly wind speed observation // Journal of Applied Meteorology and Climatology. 1991. - V. 30. - № 6. - P. 823-833. URL https://doi.org/10.1175/1520-0450(1991)030<0823:TAOHWS> 2.0.СО;2 (дата обращения: 15.03.2021).

6. Fortuna L., Nunnari G., Nunnari S. Nonlinear modeling of solar radiation and wind speed time series. - Switzerland, Cham Springer, 2016. - 98 p. URL: https://doi.org/10.1007/978-3-31938764-2 (дата обращения: 15.03.2021).

\section{Заключение}

Предложен метод моделирования скорости ветра на основе стохастического дифференциального уравнения дробного процесса Орнштейна-Уленбека с периодической функцией среднего. По сравнению с моделью процесса с некоррелированными приращениями, предложенная модель на основе дробного процесса, обладающего свойством длительной памяти, позволяет более достоверно смоделировать автокорреляционную зависимость, что характеризуется высоким показателем коэффициента детерминации, который в среднем составил $R^{2}=0,93$. Результаты анализа показали, что среднее значение отклонения оценки показателя Херста смоделированных временных рядов от фактических данных составило менее $2 \%$. Реализация алгоритма моделирования выполнена в программной среде MATLAB. Практическое применение модели нацелено на ее использование в исследованиях режимов работы ветроэнергетических установок и электроэнергетических систем на их основе.

7. Numerical investigations into the idealized diurnal cycle of atmospheric boundary layer and its impact on wind turbine's power performance / L. Tian, Y. Song, N. Zhao, W. Shen, T. Wang, C. Zhu // Renewable Energy. - 2020. - V. 145. P. 419-427. URL: https://doi.org/10.1016/j.renene.2019.05.038 (дата обращения: 15.03.2021).

8. Baker R.W., Walker S.N., Wade J.E. Annual and seasonal variations in mean wind speed and wind turbine energy production // Solar Energy. - 1990. - V. 45. - № 5. - P. 285-289. URL: https://doi.org/10.1016/0038-092X(90)90013-3 (дата обращения: 15.03.2021)

9. Seasonal variability of offshore wind turbine wakes / W. Chanprasert, R. Sharma, J. Cater, S. Norris // 22nd Australasian Fluid Mechanics Conference AFMC2020. - Brisbane, 7-10 December 2020. URL: https://doi.org/10.14264/d1247d1 (дата обращения: 15.03.2021).

10. Ghofrani M., Alolayan M. Time series and renewable energy forecasting // Time Series Analysis and Applications / Ed. by N. Mohamudally. - IntechOpen, 2018. - P. 77-92. URL: https://doi.org/10.5772/intechopen.70845 (дата обращения: 15.03.2021).

11. Bazionis I.K., Georgilakis P.S. Review of deterministic and probabilistic wind power forecasting: models, methods, and future research // Electricity. - 2021. - V. 2. - № 1. - P. 13-47. URL: 
https://doi.org/10.3390/electricity2010002 (дата обращения: 15.03.2021)

12. Elwan A.A., Habibuddin M.H. Statistical approach for wind speed forecasting using Markov chain modelling as the probabilistic model // 2019 IEEE 2nd International Conference on Power and Energy Applications (ICPEA). - Singapore, 2019. - P. 177-182 URL: http://doi.org/10.1109/ICPEA.2019.8818533 (дата обращения: 15.03.2021)

13. Zárate-Miñano R., Anghel M., Milano F. Continuous wind speed models based on stochastic differential equations // Applied Energy. - 2013. - V. 104. - P. 42-49. URL: https://doi.org/10. 1016/j.apenergy.2012.10.064 (дата обращения: 15.03.2021).

14. Arenas-López J.P., Badaoui M. The Ornstein-Uhlenbeck process for estimating wind power under a memoryless transformation / Energy. - 2020. - V. 213. - № 118842. URL: https://doi.org/ 10.1016/j.energy.2020.118842 (дата обращения: 15.03.2021).

15. Short-term probabilistic forecasting of wind speed using stochastic differential equations / E.B. Iversen, J.M. Morales, J.K. Møller, H. Madsen // International Journal of Forecasting. - 2016. V. 32. - № 3. - P. 981-990. URL: https://doi.org/10.1016/j.ijforecast. 2015.03.001 (дата обращения: 15.03.2021).

16. Ibe O.C. Markov processes for stochastic modeling. 2nd ed. Elsevier: Academic Press, 2013. - 514 p. URL: https://doi.org/ 10.1016/C2012-0-06106-6 (дата обращения: 15.03.2021).

17. Stochastic calculus for fractional Brownian motion and applications / F. Biagini, Y. Hu, B. Øksendal, T. Zhang. - London Springer, 2008. - 330 p. URL: https://doi.org/10.1007/978-184628-797-8 (дата обращения: 15.03.2021).

18. Alaton P., Djehiche B., Stillberger D. On modelling and pricing weather derivatives // Applied Mathematical Finance. - 2002. V. 9. - № 1. - P. 1-20. URL: https://doi.org/10.1080/ 13504860210132897 (дата обращения: 15.03.2021).

19. Lysy M., Pillai N.S. Statistical inference for stochastic differential equations with memory // arXiv.org. 2013. URL: https://arxiv.org/abs/1307.1164v1 (дата обращения: 15.03.2021).
20. Fast and exact synthesis for 1-D fractional Brownian motion and fractional Gaussian noises / E. Perrin, R. Harba, R. Jennane, I. Iribarren // IEEE Signal Processing letters. - 2002. - V. 9. № 11. - P. 382-384. URL: https://doi.org/10.1109/ LSP.2002.805311 (дата обращения: 15.03.2021).

21. Jung C., Schindler D. Wind speed distribution selection - a review of recent development and progress // Renewable and Sustainable Energy Reviews. - 2019. - V. 144. - 109290. URL: https://doi.org/10.1016/j.rser.2019.109290

22. Taqqu M.S., Teverovsky V., Willinger W. Estimators for longrange dependence: an empirical study // Fractals. - 1995. - V. 3. № 4. - P. 785-798. URL: https://doi.org/10.1142/S0218348X 95000692 (дата обращения: 15.03.2021)

23. Su Y., Wang Y. Parameter estimation for fractional diffusion process with discrete observations // Journal of function spaces. 2019. - 9036285. URL: https://doi.org/10.1155/2019/9036285 (дата обращения: 15.03.2021).

24. Xiao W., Zhang W., Xu W. Parameter estimation for fractional Ornstein-Uhlenbeck processes at discrete observation // Applied Mathematical Modelling. - 2011. - V. 35. - № 9. - P. 4196-4207. URL: https://doi.org/10.1016/j.apm.2011.02.047 (дата обращения: 15.03.2021).

25. Специализированные массивы для климатических исследований // Всероссийский научно исследовательский институт гидрометеорологической информации - Мировой центр данных (ВНИИГМИ-МЦД). URL: http://aisori-m.meteo.ru (дата обращения: 15.03.2021).

26. Wilks D.S. Statistical methods in the atmospheric sciences. 2nd ed. - Burlington, MA, USA: Elsevier, Academic Press, 2006. -648 p.

27. Burnham K.P., Anderson D.R. Model selection and multimodel inference: a practical information-theoretic approach. 2nd ed. New York: Springer, 2002. - 488 p. URL: https://doi.org/10.1007/b97636 (дата обращения: 15.03.2021).

Поступила 12.04.2021 2.

\section{Информация об авторах}

Давыдов Д.Ю., аспирант отделения электроэнергетики и электротехники Инженерной школы энергетики Национального исследовательского Томского политехнического университета.

Обухов С.Г., доктор технических наук, профессор отделения электроэнергетики и электротехники Инженерной школы энергетики Национального исследовательского Томского политехнического университета. 
UDC 621.311 .24

\title{
WIND SPEED MODEL BASED ON FRACTIONAL STOCHASTIC PROCESS
}

\author{
Denis Y. Davydov 1 , \\ denisyudavydov@gmail.com
}

\author{
Sergey G. Obukhov ${ }^{1}$, \\ serob99@mail.ru \\ 1 National Research Tomsk Polytechnic University,
30, Lenin avenue, Tomsk, 634050, Russia.
}

Relevance. The urgent task of the technical and economic development of the Northern and Eastern regions of Russia is to provide reliable and efficient power supply to consumers, geographically located in remote, hard-to-reach areas. The use of wind power plants is a promising way to solve this problem. The primary task of the design and feasibility study of the use of wind power plants is to predict changes in wind speeds at the site of the power plant installation. The stochastic nature of the wind and its spatiotemporal variability explain the high complexity of this problem, for the solution of which the methods of mathematical modeling are used. The known models of wind speed based on Markov chains, autoregressive moving-average and other discrete models do not allow varying the time step, which does not allow their use for simulation of operating modes of wind turbines and wind energy systems. The article proposes a model of wind speed based on the stochastic differential equation which eliminates this drawback.

The aim of the study is to construct wind speed model based on fractional Ornstein-Uhlenbeck process with a periodic long-term mean, which provides modeling of static and dynamic modes of operation of a wind power plant at different time intervals.

Methods: mathematical and computer modeling using Matlab/Simulink software environment.

Results. The proposed model, in contrast to the known SDE-based models, is able to produce autocorrelated wind speed trajectories with long-term dependence, daily and monthly variations to perform more detailed simulation of the operating modes of wind turbines at different time intervals with the required time step. The model was tested using the data of climatic observations of wind speed obtained from electronic archive of the All-Russian Institute of Hydrometeorological Information. The model adequacy was evaluated by comparing characteristics of simulated wind speed trajectories with actual observations collected at 518 weather stations located on territory of Russia.

\section{Key words:}

Wind energy, wind speed modeling, stochastic differential equations, fractional Brownian motion, time series.

\section{REFERENCES}

1. Choi Y., Lee C., Song J. Review of Renewable Energy Technologies Utilized in the Oil and Gas Industry. International Journal of Renewable Energy Research, 2017, vol. 7, no. 2, pp. 592-598.

2. Belskiy A.A., Klimko V.I. Oil production intensification. The concept of the thermal method with the use of autonomous wind power plants. Delovoy zhurnal «Neftegaz.RU», 2016, no. 1-2, pp. 38-41. In Rus.

3. Goryunov O.A., Nazarova Yu.A. Prospects for wind driven power plants application for power supply to the gas industry facilities in the Far North regions. Territoriya «NEFTEGAZ», 2015, no. 12, pp. 146-150. In Rus.

4. Vasenin A.B., Kryukov O.V. Voprosy elektropitaniya vdoltrassovykh obektov Edinoy sistemy gazosnabzheniya Rossii [Power supply issues for along-route facilities of the Unified Gas Supply System of Russia]. Nauchno-tekhnicheskiy sbornik «Vesti gazovoy nauki», 2020, no. 2 (44), pp. 181-192.

5. Brett A.C., Tuller S.E. The autocorrelation of hourly wind speed observation. Journal of Applied Meteorology and Climatology, 1991, vol. 30, no. 6, pp. 823-833. Available at: https://doi.org/ 10.1175/1520-0450(1991)030<0823:TAOHWS >2.0.CO;2 (accessed 15 March 2021)

6. Fortuna L., Nunnari G., Nunnari S. Nonlinear modeling of solar radiation and wind speed time series. Cham, Springer, 2016. 98 p. Available at: https://doi.org/10.1007/978-3-319-38764-2 (accessed 15 March 2021).

7. Tian L., Song Y., Zhao N., Shen W., Wang T., Zhu C. Numerical investigations into the idealized diurnal cycle of atmospheric boundary layer and its impact on wind turbine's power performance. Renewable Energy, 2020, vol. 145, pp. 419-427. Available at: https://doi.org/10.1016/j.renene.2019.05.038

8. Baker R.W., Walker S.N., Wade J.E. Annual and seasonal variations in mean wind speed and wind turbine energy production. Solar Energy, 1990, vol. 45, no. 5, pp. 285-289. Available at: https://doi.org/10.1016/0038-092X(90)90013-3
9. Chanprasert W., Sharma R., Cater J., Norris S. Seasonal variability of offshore wind turbine wakes. 22 $2^{\text {nd }}$ Australasian Fluid Mechanics Conference AFMC2020. Brisbane, 2020, 7-10 December. Available at: https://doi.org/10.14264/d1247d1 (accessed 15 March 2021).

10. Ghofrani M., Alolayan M. Time series and renewable energy forecasting. Time Series Analysis and Applications. Ed. by N. Mohamudally. IntechOpen, 2018. pp. 77-92. Available at: https:// doi.org/10.5772/intechopen.70845 (accessed 15 March 2021).

11. Bazionis I.K., Georgilakis P.S. Review of deterministic and probabilistic wind power forecasting: models, methods, and future research. Electricity, 2021, vol. 2, no. 1, pp. 13-47. Available at: https://doi.org/10.3390/electricity2010002 (accessed 15 March 2021).

12. Elwan A.A., Habibuddin M.H. Statistical approach for wind speed forecasting using Markov chain modelling as the probabilistic model. 2019 IEEE $2^{\text {nd }}$ International Conference on Power and Energy Applications (ICPEA). Singapore, 2019. pp. 177-182. Available at: http://doi.org/10.1109/ICPEA.2019.8818533 (accessed 15 March 2021)

13. Zárate-Miñano R., Anghel M., Milano F. Continuous wind speed models based on stochastic differential equations. Applied Energy, 2013, vol. 104, pp. 42-49. Available at: https://doi.org/ 10.1016/j.apenergy.2012.10.064 (accessed 15 March 2021).

14. Arenas-López J.P., Badaoui M. The Ornstein-Uhlenbeck process for estimating wind power under a memoryless transformation. Energy, 2020, vol. 213, no. 118842. Available at: https://doi.org/10.1016/j.energy.2020.118842 (accessed 15 March 2021).

15. Iversen E.B., Morales J.M., Møller J.K., Madsen H. Short-term probabilistic forecasting of wind speed using stochastic differential equations. International Journal of Forecasting, 2016, vol. 32, no. 3, pp. 981-990. Available at: https://doi.org/10.1016/ j.ijforecast.2015.03.001 (accessed 15 March 2021).

16. Ibe O.C. Markov processes for stochastic modeling. $2^{\text {nd }}$ ed. Elsevier, Academic Press, 2013. 514 p. Available at: 
https://doi.org/ 10.1016/C2012-0-06106-6 (accessed 15 March 2021).

17. Biagini F., Hu Y., Øksendal B., Zhang T. Stochastic calculus for fractional Brownian motion and applications. London, Springer, 2008. 330 p. Available at: https://doi.org/10.1007/978-1-84628797-8 (accessed 15 March 2021)

18. Lysy M., Pillai N.S. Statistical inference for stochastic differential equations with memory. Available at: https://arxiv.org/ abs/1307.1164v1 (accessed: 15 March 2021).

19. Alaton P., Djehiche B., Stillberger D. On modelling and pricing weather derivatives. Applied Mathematical Finance, 2002, vol. 9, no. 1, pp. 1-20. Available at: http://dx.doi.org/10.1080/ 13504860210132897 (accessed: 15 March 2021).

20. Perrin E., Harba R., Jennane R., Iribarren I. Fast and exact synthesis for 1-D fractional Brownian motion and fractional Gaussian noises IEEE Signal Processing letters, 2002, vol. 9, no. 11, pp. 382-384. Available at: https://doi.org/10.1109/LSP.2002.805311 (accessed 15 March 2021).

21. Jung C., Schindler D. Wind speed distribution selection - a review of recent development and progress. Renewable and Sustainable Energy Reviews, 2019, vol. 114, 109290. Available at: https://doi.org/10.1016/j.rser.2019.109290 (accessed: 15 March 2021).

22. Taqqu M.S., Teverovsky V., Willinger W. Estimators for long-range dependence: an empirical study. Fractals, 1995, vol. 3, no. 4 pp. 785-798. Available at: https://doi.org/10.1142/S0218348X 95000692 (accessed: 15 March 2021).

23. Su Y., Wang Y. Parameter estimation for fractional diffusion process with discrete observations. Journal of function spaces, 2019, $9036285 . \quad$ Available at: https://doi.org/10.1155/2019/9036285

24. Xiao W., Zhang W., Xu W. Parameter estimation for fractional Ornstein-Uhlenbeck processes at discrete observation. Applied Mathematical Modelling, 2011, vol. 35, no. 9, pp. 4196-4207. Available at: https://doi.org/10.1016/j.apm.2011.02.047 (accessed: 15 March 2021).

25. Specializirovannye massivy dlya klimaticheskikh issledovaniy. Vserossiyskiy nauchno issledovatelskiy institut gidrometeorologicheskoy informatsii - Mirovoy tsentr dannykh (VNIIGMI-MCD) [Specialized arrays for climatic research. All-Russian Research Institute of Hydrometeorological Information (RIHMI-WDC)]. Available at: http://aisori-m.meteo.ru (accessed 15 March 2021).

26. Wilks D.S. Statistical methods in the atmospheric sciences. $2^{\text {nd }}$ ed. Burlington, MA, USA, Elsevier, 2006. 649 p.

27. Burnham K.P., Anderson D.R. Model selection and multimodel inference: a practical information-theoretic approach. $2^{\text {nd }}$ ed. New York, Springer, 2002. 488 p. Available at: https://doi.org/ 10.1007/b97636 (accessed 15 March 2021).

Received: 12 April 2021.

\section{Information about the authors}

Denis Y. Davydov, postgraduate student, National Research Tomsk Polytechnic University.

Sergey G. Obukhov, Dr. Sc., professor, National Research Tomsk Polytechnic University. 